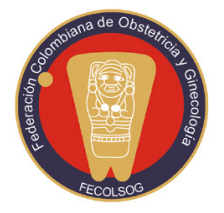

Revista Colombiana de Obstetricia y Ginecología Vol. 69 No. 1 • Enero-Marzo 2018 • (32-41)

\title{
ASOCIACIÓN ENTRE FACTORES SOCIOAMBIENTALES Y LA PRÁCTICA DE ACTIVIDAD FÍSICA EN MUJERES EMBARAZADAS: ESTUDIO DE CORTE TRANSVERSAL
}

\author{
Association between socio-envirommental factors \\ and the practice of physical activity in pregnant \\ women: A cross-sectional study \\ Luzdaris De Ávila-Quintana ${ }^{1}$, Sindy Ariza-Egea², Boris Llanos-González ${ }^{3}$, \\ Yaneth Herazo-Beltrán ${ }^{4}$, Regina Domínguez-Anaya ${ }^{5}$ \\ Recibido: abril 27/17 - Aceptado: marzo 8/18
}

\section{RESUMEN}

Objetivo: establecer la asociación entre factores socioambientales y la práctica de actividad física en mujeres embarazadas.

Materiales y métodos: estudio de corte transversal realizado en mujeres gestantes sin contraindicaciones para realizar actividad física, que asistían a la consulta prenatal, pertenecientes a dos instituciones de salud de la ciudad de Barranquilla y otra de la ciudad de Cartagena de Indias, Colombia. El muestreo fue intencional. Se evaluaron las características sociodemográficas, los niveles de actividad física y la percepción de factores socioambientales como infraestructura del barrio, las cualidades estéticas,

1 Fisioterapeuta; Magíster en Actividad Física y Salud, Programa de Fisioterapia, Universidad San Buenaventura, Cartagena (Colombia). deavilaquintana@gmail.com

2 Fisioterapeuta; Magíster en Actividad Física y Salud, Programa de Fisioterapia, Universidad del Sinú, Montería (Colombia).

3 Fisioterapeuta; Magíster en Actividad Física y Salud, Cartagena (Colombia)

4 Fisioterapeuta; Magíster en Salud Pública, Facultad de Ciencias de la Salud, Universidad Simón Bolívar, Barranquilla (Colombia).

5 Especialista en Epidemiología, Programa de Fisioterapia, Universidad San Buenaventura, Cartagena (Colombia). el ambiente social, la conexión entre las calles, la seguridad y tráfico en el barrio. Se estimó la razón de odds (OR).

Resultados: de un total de 228 mujeres gestantes se incluyeron 208 en el análisis. El 62\% de las gestantes no cumplen las recomendaciones de actividad física para mantener su salud. Entre los factores socioambientales relacionados con la inactividad física de las embarazadas están el residir a mucha distancia de tiendas y supermercados para ir caminando $(\mathrm{OR}=5,1$; IC $95 \%$ : 2,8-9,4); presencia de aceras en mal estado en las calles $(\mathrm{OR}=3,4$; IC $95 \%$ : 1,9-6,3); pocas personas físicamente activas en el barrio (OR = 4,1; IC $95 \%$ : 2,2-7,5); inseguridad en el día y en la noche para caminar $(\mathrm{OR}=3,6$; $\mathrm{IC}$ $95 \%: 1,9-6,4)$.

Conclusión: la percepción de las características socioambientales está asociada a la actividad física de las gestantes. Se requieren intervenciones que mejoren las características socioambientales que afectan la percepción de estos factores en la población, para evaluar su efecto en la actividad física de las gestantes. 
Palabras clave: actividad motora, ambiente, embarazo.

\section{ABSTRACT}

Objective: To establish the relationship between social and environmental determinants and the practice of physical activity in pregnant women.

Materials and methods: Cross-section study conducted in pregnant women with no contraindication to physical activity; these women came for prenatal consultation and were affiliated to two healthcare providers in Barranquilla and Cartagena de Indias, Colombia. Sampling was intentional. The social and demographic characteristics were evaluated, as well as the levels of physical activity and the perception of socio-environmental factors, including neighborhood infrastructure, esthetic considerations, the social environment, road connectivity, safety and surrounding traffic conditions. The raw Odds Ratio (OR) was estimated.

Results: Out of 228 pregnant women, 208 were included in the analysis; $62 \%$ of the pregnant women fail to comply with the physical activity recommendations for maintaining their health. Some of the socio-environmental factors associated with a sedentary lifestyle in pregnancy are: living far away to walk to shops and supermarkets $(\mathrm{OR}=5.1 ; 95 \%$ CI: 2.8-9.4); poor sidewalk conditions $(\mathrm{OR}=3.4$; 95\% CI: 1.9-6.3); few people exercising around the neighborhood (OR $=4.1 ; 95 \%$ CI: 2.2-7.5); not safe to walk during the day or evening $(\mathrm{OR}=3.6 ; 95 \%$ CI: 1.9-6.4).

Conclusion: The perception of the socio-environmental characteristics is associated with physical activity in pregnant women. Interventions are needed to improve the socio-environmental characteristics that impact the perception of the population about these factors, in order to assess its impact on the physical activity of pregnant women.

Key words: Motor activity, environment, pregnancy.

\section{INTRODUCCIÓN}

La actividad física (AF) ha sido definida por la Organización Mundial de la Salud (OMS) como cualquier movimiento corporal producido por los músculos esqueléticos que exija un gasto energético (1). Durante la gestación, la actividad física adquiere peculiar consideración por tratarse de un periodo en el que se producen diversas modificaciones anatomo-fisiológicas y psíquicas que requieren de la embarazada una adaptación continua hacia la actividad (2). De acuerdo con las recomendaciones de la OMS (3) sobre AF, su práctica con regularidad proporciona beneficios para la salud en todas las edades de la vida. Cabe resaltar que durante el periodo de gestación la AF es de gran importancia por los múltiples beneficios que aporta, tanto a la salud materna como a la del niño, incluyendo la prevención y el control de la diabetes gestacional, control del aumento excesivo de peso, reducción del dolor de espalda y pélvico, disminución de la depresión durante el embarazo y después del parto, entre otros $(2,4)$.

A pesar de las recomendaciones del Colegio Americano de Obstetras y Ginecólogos (ACOG) sobre la práctica segura de AF, al menos 150 minutos por semana de actividad aeróbica de intensidad moderada (5), son pocas las gestantes que logran las recomendaciones mínimas de actividad física en el transcurso del embarazo; varios estudios en diferentes países reportan bajas frecuencias de AF en las gestantes; en Chile, solo el 20,3\% son suficientemente activas (6); en Estados Unidos, el $70 \%$ informaron que no participan en AF moderada y vigorosa (7), mientras en que China, solo el 11,1\% cumplieron con la guía de actividad física durante el embarazo (8).

Los estudios sobre AF en la mujer embarazada reportan el uso de diferentes cuestionarios para su medición (6), uno de ellos es el Cuestionario Internacional de Actividad Física (IPAQ), que en su versión corta incluye preguntas respecto a la evaluación de la actividad física al caminar, o si se ha realizado de manera moderada o vigorosa, así como el comportamiento sedentario, y permite categorizar a los sujetos de estudio como: inactivos, regularmente activos y muy activos; este cuestionario ha sido validado en varios países, incluidos 
algunos de América Latina y Centroamérica, y ha mostrado una adecuada confiabilidad (8, 9-11).

Los bajos niveles de AF en las embarazadas podrían ser explicados mediante el modelo ecológico y los determinantes socioambientales; el primero propone la interrelación de factores individuales y ambientales como los determinantes de un estilo de vida físicamente activo, es decir, el entorno del barrio donde residen las personas contribuye a la adquisición de comportamientos saludables, de allí que la presencia de aceras bien construidas, poco tráfico y seguridad en el barrio, entre otros, faciliten e incrementen el transporte activo. El modelo ecológico orienta las intervenciones de promoción de comportamientos saludables desde la inclusión de factores intrapersonales, interpersonales, institucionales y comunitarios, destacando la necesidad de realizar cambios en los entornos sociales y físicos para el cambio de comportamiento individual (12, 13). Los determinantes socioambientales hacen referencia al ambiente físico y social de los entornos donde transcurre la vida de las personas que pueden facilitar o limitar una adecuada y segura práctica de AF; en este sentido, paisajes agradables, otras personas que realicen AF, seguridad y zonas verdes próximas son factores que se asocian de manera positiva con un estilo de vida activo (14). El ambiente físico mal construido se ha asociado con el parto prematuro, la ganancia de peso durante el embarazo y el bajo peso al nacer del niño $(15,16)$.

Down et al. consideran que comprender los efectos de los factores ambientales que motivan o impiden que las mujeres embarazadas participen en la AF es un imperativo porque guían la planificación e implementación de las intervenciones, desde un marco socioecológico de múltiples niveles (17). Son pocos los estudios en Colombia que explican la influencia de los factores socioambientales en la práctica de AF durante el embarazo, por ello, los objetivos del presente estudio fueron establecer los niveles de actividad física y su asociación con la percepción de factores socioambientales en un grupo de mujeres embarazadas.

\section{MATERIALES Y MÉTODOS}

Estudio de corte transversal, analítico, en mujeres embarazadas que asistían a la consulta prenatal sin contraindicaciones para realizar actividad física y que aceptaran voluntariamente participar en el estudio. Ellas fueron seleccionadas, entre los meses de enero y abril de 2016, de instituciones de salud de primer nivel de atención, localizadas en las ciudades de Barranquilla y Cartagena de Indias, en la costa Caribe de Colombia. Ambas instituciones atienden población afiliada al régimen subsidiado por el Estado en el sistema de seguridad social en Colombia. El muestreo fue no probabilístico e intencional.

Procedimiento. En la sala de espera de cada institución se buscaron las gestantes que cumplieran con los criterios de selección. En aquellas que aceptaron participar, y previa firma del consentimiento informado, se aplicaron los instrumentos de evaluación por los investigadores con experiencia en la medición de las variables de estudio: una encuesta que indagó sobre las características sociodemográficas de las gestantes, y el Cuestionario Internacional de Actividad Física (IPAQ formato corto) para medir el nivel de actividad física, tomando como referencia para su clasificación la guía del IPAQ (18). Para medir la percepción del ambiente social y físico del barrio se utilizó el Módulo Ambiente del International Physical Activity Study (IPAS), el cual consta de dieciséis preguntas sobre los factores ambientales del barrio; las preguntas del módulo se basan en una escala tipo Likert de 4 puntos: muy en desacuerdo, en desacuerdo, de acuerdo y muy de acuerdo. El instrumento ha sido usado en otros estudios (13) y se han reportado coeficientes de correlación intraclase entre 0,43 a 0,91 (19). El IPAS permitió evaluar las características socioambientales y las cualidades estéticas del barrio.

Se midieron la edad, el estrato socioeconómico, el estado civil, la ocupación laboral y el nivel educativo. El IPAQ permitió categorizar a las gestantes en niveles de AF alto, medio y bajo, según si cumplían la recomendación de 150 minutos de AF moderada durante la semana; también permitió estimar el comportamiento sedentario en $\mathrm{min} /$ día y $\mathrm{min} / \mathrm{se}$ - 
manal, y el gasto energético expresado en Metabolic Equivalent of Task (MET), que corresponde a la unidad de medida del índice metabólico. De acuerdo con las recomendaciones actuales se consideró una gestante activa físicamente la que alcanzó un gasto energético mayor de 450 MET por semana; el punto de corte de 450 MET se definió a partir de la multiplicación del consumo mínimo de 3 MET de actividad física moderada por los 150 minutos semanales que debe cumplir una embarazada para ser activa físicamente. Como características socioambientales del barrio se midieron: presencia de aceras en las calles, instalaciones para bicicleta en el vecindario, instalaciones recreativas en el vecindario, nivel de tráfico para caminar o andar en bicicleta, presencia de personas físicamente activas en el barrio y percepción de seguridad para caminar o andar en bicicleta. La variable de desenlace fue la práctica de $\mathrm{AF}$, cuyas categorías fueron: activas e inactivas; se consideró una gestante activa la que fue categorizada en los niveles alto y medio; e inactiva, la clasificada en el nivel bajo de AF. Un nivel alto lo lograron las embarazadas que realizan más de 300 minutos a la semana de AF de intensidad moderada; un nivel medio, las que realizan entre 150 y 300 minutos de AF de intensidad moderada a la semana; y un nivel bajo, las que realizan menos de 150 minutos a la semana.

Análisis estadístico. Por medio del programa estadístico SPSS versión 24, se estableció la frecuencia de las variables categóricas: características sociodemográficas y los niveles de AF; se realizó la prueba de Kolmogórov-Smirnov (K-S) para verificar la distribución de los datos de las variables continuas: gasto energético y comportamiento sedentario de las participantes, y definir el tipo de medidas de tendencia central y de dispersión. Para identificar los factores socioambientales asociados con el nivel de AF (activo/inactivo) se realizó una regresión logística bivariada mediante la cual se determinaron los Odd Ratio (OR), y su intervalo de confianza (IC) del $95 \%$. En el modelo fue considerada como variable de exposición la percepción de las características socioambientales del barrio y como variable de desenlace la práctica de AF.

El estudio fue aprobado por el Comité de Ética de la Universidad Simón Bolívar de Barranquilla. La financiación de este proyecto fue asumida por los investigadores y la Universidad Simón Bolívar de Barranquilla.

\section{RESULTADOS}

La población total de gestantes estuvo constituida por 228 mujeres, de estas se descartaron 20 (5 no diligenciaron de manera completa las encuestas y 15 tenían recomendación médica para no realizar ejercicio físico). De ellas, 119 pertenecían a las IPS de Barranquilla. La edad media de las gestantes fue 23,6 \pm 6,51 años, el 66,3\% se encontraban en un rango de edad entre los 18 y 29 años. La tabla 1 muestra las características sociodemográficas de las gestantes; el 71,2 \% eran de estrato socioeconómico 1, el 65,9\% vivía en unión libre, el 31,3\% informó un nivel educativo de secundaria completa y el 84,6\% estaban desempleadas.

En cuanto al nivel de actividad física, el $62 \%$ de las embarazadas tiene un nivel bajo de AF, 22,6\% un nivel alto y el 15,4\% un nivel medio; se observó 0 minutos de actividad física vigorosa y, por tanto, no hubo equivalentes metabólicos (MET) de ejercicio vigoroso; el tipo de actividad física que más le aporta al consumo energético es caminar (156,7 MET). Lo anterior coincide con un mayor número de minutos semanales caminando (47,5 min); al menos el $50 \%$ de las pacientes informaron el comportamiento sedentario con más de 6 horas al día sentadas o acostadas (tabla 2).

La tabla 3 indica una relación entre los niveles de actividad física y los determinantes socioambientales; se encontraron como factores de riesgo para una actividad física baja el residir a mucha distancia de tiendas y supermercados para ir caminando o en bicicleta (OR = 5,1; IC $95 \%: 2,8-9,4)$; la presencia de aceras en mal estado en las calles $(\mathrm{OR}=3,4$; $\mathrm{IC}$ $95 \%: 1,9-6,3)$; percibir pocas personas físicamente activas en el barrio (OR = 4,1; IC $95 \%$ : 2,2-7,5); e 


\begin{tabular}{|c|c|c|}
\hline Rango de edad & $\mathrm{n}=\mathbf{2 0 8}$ & $(\%)$ \\
\hline 12-17 años & 29 & 13,9 \\
\hline 18-29 años & 138 & 66,3 \\
\hline 30-39 años & 41 & 19,7 \\
\hline \multicolumn{3}{|l|}{ Estrato socioeconómico } \\
\hline Estrato 1 & 148 & 71,2 \\
\hline Estrato 2 & 47 & 22,6 \\
\hline Estrato 3 & 12 & 5,8 \\
\hline Estrato 4 & 1 & 0,5 \\
\hline \multicolumn{3}{|l|}{ Estado civil } \\
\hline Soltera & 40 & 19,2 \\
\hline Casada & 28 & 13,5 \\
\hline Unión libre & 137 & 65,9 \\
\hline Separada/Divorciada & 2 & 1,0 \\
\hline Viuda & 1 & 0,5 \\
\hline \multicolumn{3}{|l|}{ Nivel educativo } \\
\hline Primaria incompleta & 7 & 3,4 \\
\hline Primaria completa & 14 & 6,7 \\
\hline Secundaria completa & 65 & 31,3 \\
\hline Secundaria incompleta & 52 & 25,0 \\
\hline Técnico/Tecnológico & 35 & 16,8 \\
\hline Estudios superiores & 24 & 11,5 \\
\hline Posgrado & 11 & 5,3 \\
\hline \multicolumn{3}{|l|}{ Ocupación } \\
\hline Empleada & 32 & 15,4 \\
\hline Desempleada & 176 & 84,6 \\
\hline
\end{tabular}

inseguridad en el barrio durante el día y la noche para caminar o andar en bicicleta $(\mathrm{OR}=3,6$; $\mathrm{IC}$ $95 \%: 1,9-6,4)$.

\section{DISCUSIÓN}

Nuestros resultados muestran que más del $60 \%$ de las gestantes participantes no cumplen las recomendaciones de expertos de al menos 150 minutos de AF aeróbica de intensidad moderada, preferiblemente, la caminata rápida $(3,20)$. El comportamiento sedentario es alto, la mitad de las embarazadas pasan más de 6 horas sentadas o acostadas. Se encontró una asociación significativa entre algunas características socioambientales del barrio y 


\begin{tabular}{|c|c|c|}
\hline Niveles de actividad física & $\begin{array}{c}n=208 \\
\text { Frecuencia }\end{array}$ & Porcentaje \\
\hline Alto & 47 & 22,6 \\
\hline Medio & 32 & 15,4 \\
\hline Bajo & 129 & 62 \\
\hline Consumo gasto energético (MET) & Mediana & Rango intercuartil \\
\hline MET actividades moderadas & 32 & 360 \\
\hline MET actividad caminar & 156,7 & 280,5 \\
\hline MET/semanal & 114,5 & 231 \\
\hline Min/Semana de actividad física & Mediana & Rango intercuartil \\
\hline Total min/semana actividades moderadas & 8 & 90 \\
\hline Total min/semana actividad caminar & 47,5 & 85 \\
\hline Total min/semanal & 107 & 225 \\
\hline Comportamiento sedentario & Mediana & Rango intercuartil \\
\hline Tiempo sentado al día & $360 \min$ (6 horas) & 240 \\
\hline Tiempo sentado semanal & $2520 \min$ (42 horas) & 1680 \\
\hline
\end{tabular}

los bajos niveles AF de las embarazadas, entre estas: pocos amigos, vecinos y familia que realizan AF en el barrio, baja seguridad para caminar, poca presencia de aceras en las calles y pocas tiendas y supermercados cercanos a las viviendas de las mujeres.

Nuestros hallazgos coinciden con lo encontrado en otros estudios en cuanto a que las mujeres tienden a reducir en nivel, duración e intensidad la actividad física durante el embarazo; en el estudio de Lindqvist (21) se reporta que el 52,9\% no logró el nivel recomendado de actividad física. En relación con los minutos de actividad física acumulados durante la semana, nuestras gestantes muestran un nivel muy inferior al descrito en el trabajo de Leppe et al. (6), en el que las gestantes acumularon en promedio 154 minutos a la semana, el 64,4\% indicó realizar AF de intensidad vigorosa, y un $22 \%$ de AF moderada.
Respecto al comportamiento sedentario nuestras observaciones muestran un mayor comportamiento sedentario al informado por Hawkins et al. (22), quienes compararon dicho comportamiento en mujeres no embarazadas y embarazadas, y encontraron que en estas últimas el tiempo sedentario fue más alto, acumulándose en episodios $\geq 30 \mathrm{~min}$ durante el día.

En este mismo sentido se observa que el consumo energético y el número de minutos semanal de la $\mathrm{AF}$ caminar es mayor que para otra actividad moderada en el hogar, trabajo o tiempo libre, resultado que es favorable toda vez que caminar ha sido reportada como una práctica saludable, gratuita y accesible durante el tiempo de ocio o como medio de transporte de las personas, y donde el ambiente de las ciudades cobra importancia por cuanto aspectos como la proximidad y la diversidad de tiendas, servicios, luga- 
Tabla 3.

Determinantes socioambientales para la realización de la actividad física en mujeres gestantes en Barranquilla y Cartagena, Colombia, 2016

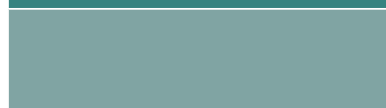

\section{Población general $n=208$}

\begin{tabular}{c|c} 
Inactivas & Activas \\
$\mathrm{n}=129$ & $\mathrm{n}=79$
\end{tabular}

OR; IC $95 \%$

Muchos lugares como tiendas y supermercados para ir a poca distancia

\begin{tabular}{|c|c|c|c|c|}
\hline Desacuerdo & $116(55,8 \%)$ & $91(78,4 \%)$ & 25 (21,6\%) & $5,1(2,8-9,4)$ \\
\hline Acuerdo & 92 (44,2 \%) & $38(41,3 \%)$ & 54 (58,7\%) & \\
\hline \multicolumn{5}{|c|}{ Presencia de aceras en las calles } \\
\hline Desacuerdo & $73(35,1 \%)$ & $48(65,8 \%)$ & $25(34,2 \%)$ & $3,4(1,9-6,3)$ \\
\hline Acuerdo & $135(64,9 \%)$ & $48(35,6 \%)$ & $87(64,4 \%)$ & \\
\hline \multicolumn{5}{|c|}{ Instalaciones para bicicleta en el vecindario } \\
\hline Desacuerdo & $161(77,4 \%)$ & $99(61,5 \%)$ & $62(38,5 \%)$ & $0,90(0,4-1,7)$ \\
\hline Acuerdo & $47(22,1 \%)$ & $30(18,6 \%)$ & $17(81,4 \%)$ & \\
\hline \multicolumn{5}{|c|}{ Instalaciones recreativas en el vecindario } \\
\hline Desacuerdo & $130(62,5 \%)$ & $79(60,8 \%)$ & $51(39,2 \%)$ & $0,9(0,5-1,5)$ \\
\hline Acuerdo & $78(37,5 \%)$ & $50(64,1 \%)$ & $28(35,9 \%)$ & \\
\hline \multicolumn{5}{|c|}{ Poco tráfico para caminar o andar en bicicleta } \\
\hline Desacuerdo & $109(52,4 \%)$ & $70(64,2 \%)$ & $36(35,8 \%)$ & $1,4(0,8-2,5)$ \\
\hline Acuerdo & $99(47,6 \%)$ & $59(59,6 \%)$ & $43(40,4 \%)$ & \\
\hline \multicolumn{5}{|c|}{ Personas físicamente activas en el barrio } \\
\hline Desacuerdo & $95(45,7 \%)$ & $75(78,9 \%)$ & $20(21,1 \%)$ & $4,1(2,2-7,5)$ \\
\hline Acuerdo & $113(54,3 \%)$ & $54(47,8 \%)$ & $59(52,2 \%)$ & \\
\hline \multicolumn{5}{|c|}{ Seguridad para caminar o andar en bicicleta } \\
\hline Desacuerdo & $128(61,5 \%)$ & $94(73,4 \%)$ & $34(26,6 \%)$ & $3,6(1,9-6,4)$ \\
\hline Acuerdo & $80(38,5 \%)$ & 35 (27,3\%) & 45 (72,7\%) & \\
\hline
\end{tabular}

res de trabajo, escuelas y espacios verdes se convierten en facilitadores o barreras para caminar como una forma de AF que contribuye a la salud y calidad de vida de las gestantes; otros estudios confirman que la percepción de un ambiente social o físico óptimo es esencial para caminar como actividad de recreación o como una forma de transporte $(23,24)$.

Nuestros hallazgos sobre el nivel de actividad física podrían tener relación con el tipo de población incluida, proveniente de nivel socioeconómico bajo. Se ha descrito que en población de bajos recursos es menor la posibilidad de encontrar factores de la comunidad que apoyen conductas saludables positivas (25). Esta población tiene menos acceso a ambientes con parques en buen estado y espacios verdes para realizar AF, los cuales brindan mayor oportunidad de contactos sociales que posibilitan aprendizajes de comportamientos preventivos en 
pro de la construcción de hábitos saludable (25). En cuanto a los determinantes socioambientales, en un estudio realizado en Estados Unidos se describe una correlación positiva entre caminar y los atributos de la comunidad, como las aceras en buen estado, sin embargo, se encontró una relación inversa con intersecciones de muchas vías (26).

En este estudio se observó que la percepción de tiendas y supermercados a poca distancia de su residencia o lugar de trabajo permitía que la gestante pudiera caminar con mayor frecuencia; lo anterior coincide con el estudio de Laraia et al. (16) donde se reporta que las mujeres que residen a mucha distancia $(4 \mathrm{~km})$ de los supermercados tuvieron mayor probabilidad de aumento de peso, posiblemente porque a mayor distancia de los lugares donde cotidianamente las personas realizan actividades diarias, es mayor el uso de transporte motorizado y poco el de transporte activo, como caminar o usar la bicicleta. En relación con la inseguridad, que en mayor medida perciben las embarazadas inactivas físicamente, también es un factor que ha sido estudiado por otros autores $(16,27)$, los cuales reportan que el temor a la delincuencia genera un estrés asociado en la gestante evitando el acceso a los espacios abiertos de recreación como parques, plazas o vías peatonales para caminar; por lo anterior, la percepción sobre la seguridad del vecindario es un elemento importante de información para dar recomendaciones sobre actividad física durante el embarazo.

La principal limitación del presente estudio fue la selección no aleatoria de la muestra, lo cual disminuye la generalización de los resultados; otra de las limitaciones es la naturaleza transversal del diseño, lo cual no permite establecer asociaciones causales toda vez que no cumple con el criterio de temporalidad. Como fortaleza se encuentra que es un estudio innovador en el país y la región.

\section{CONCLUSIONES}

Se concluye que la mayoría de las embarazadas participantes tienen bajos niveles de actividad física, que están relacionados con factores socioambientales como residir a mucha distancia de tiendas y supermercados para ir caminando o en bicicleta; presencia de aceras en mal estado en las calles; percibir pocas personas físicamente activas en el barrio; e inseguridad en el barrio durante el día y la noche para caminar o andar en bicicleta. Por ello, es un imperativo la implementación de estrategias multinivel de promoción de la salud dirigidas a lograr prácticas saludables permanentes en las gestantes.

\section{REFERENCIAS}

1. Organización Mundial de la Salud. 57ª Asamblea mundial de la salud. Estrategia mundial sobre régimen alimentario, actividad física y salud. Ginebra: OMS; 2004.

2. Aguilar CM, Sánchez LA, Rodríguez BR, Noack SJ, Pozo CM, López CG, et al. Physical activity by pregnant women and its influence on maternal and foetal parameters; a systematic review. Nutr Hosp. 2014;30:719-26.

3. Organización Mundial de la Salud. Recomendaciones mundiales sobre actividad física para la salud. Ginebra: OMS; 2010.

4. Nascimento SL, Surita FG, Cecatti JG. Physical exercise during pregnancy: A systematic review. Curr Opin Obstet Gynecol. 2007;24:387-94. https://doi. org/10.1097/GCO.0b013e328359f131

5. American College of Obstetricians and Gynecologists (ACOG). ACOG Committee Opinion No. 650: Physical activity and exercise during pregnancy and the postpartum period. Obstet Gynecol. 2015;126:e135-42. https://doi.org/10.1097/AOG.0000000000001214

6. Leppe ZJ, Besomi MM, Olsen CC, Mena IM, Roa S. Nivel de actividad física según GPAQ en mujeres embarazadas y postparto que asisten a un centro de salud familiar. Rev Chil Obstet Ginecol. 2013;78:425-31. https://doi.org/10.4067/S071775262013000600004

7. Hesketh KR, Evenson KR. Prevalence of U.S. Pregnant Women Meeting 2015 ACOG Physical Activity Guidelines. Am J Prev Med. 2016;51:87-9. https:// doi.org/10.1016/j.amepre.2016.05.023

8. Zhang Y, Dong S, Zuo J, Hu X, Zhang H, Zhao Y. Physical activity level of urban pregnant women 
in Tianjin, China: A cross-sectional study. PLoS One 2014; 9:1-8. https://doi.org/10.1371/journal. pone. 0109624

9. Hallal P, Gómez L, Parra D, Lobelo F, Mosquera J, Florindo A, et al. Lecciones aprendidas después de 10 Años del uso de IPAQ en Brasil y Colombia. J Phys Act Health. 2010;7:259-S264. https:// doi.org/10.1123/jpah.7.s2.s259

10. Craig CL, Marshal AL, Sjostrom M, Bauman AE, Booth ML, Ainsworth BE, et al. International physical activity questionnaire: 12-country reliability and validity. Med Sci Sports Exerc. 2003;35:1381-95. https://doi. org/10.1249/01.MSS.0000078924.61453.FB

11. Rêgo AS, Alves MT, Batista RF, Ribeiro CC, Bettiol H, Cardoso VC, et al. Physical activity in pregnancy and adverse birth outcomes. Cad Saúde Pública 2016;32:110. https://doi.org/10.1590/0102-311x00086915

12. Sallis JF, Cervero RB, Ascher W, Henderson KA, Kraft MK, Kerr J. An ecological approach to creating active living communities. Annu Rev Public Health. 2006; 27:297-322. https://doi.org/10.1146/annurev. publhealth.27.021405.102100

13. Herazo BY, Pinillos PY, Vidarte CJ, Suarez PD, Crissien QE. Perception of the environment for walking according the locality in Barranquilla, Colombia. Rev Int Med Cienc Act Fís Deporte. 2017;17:153-66.

14. Lozada AL, Campero L, Hernández B, Rubalcava L, Neufeld LM. Barreras y facilitadores para actividad física durante el embarazo y posparto en mujeres pobres de México. Salud Pública Méx. 2015;57:24251. https://doi.org/10.21149/spm.v57i3.7563

15. Miranda ML, Messer LC, Kroeger GL. Associations between the quality of the residential built environment and pregnancy outcomes among women in North Carolina. Environ Health Perspect. 2012;120:471-7.

16. Laraia B, Messer LC, Evenson K. Neighborhood factors associated with physical activity and adequacy of weight gain during pregnancy. J Urban Health. 2007;84:793-806. https://doi.org/10.1007/s11524007-9217-z

17. Downs DS, Chasan-Taber L, Evenson KR, Leiferman J, Yeo S. Physical activity and pregnancy: Past and present evidence and future recommendations. Res Q Exerc
Sport 2012;83:485-502. https://doi.org/10.1080/02 701367.2012.10599138

18. International Physical Activity Questionnaire. Guidelines for data processing and analysis of the International Physical Activity Questionnaire (IPAQ), in Short and Long Forms; 2005. p. 1-15. Disponible en www.ipaq.ki.se

19. Oyeyemi A, Adegoke B, Oyeyemi AY, Fatudimu B. Test-retest reliability of IPAQ environmental- module in an African population. Int J Behav Nutr Phys Act. 2008;5:1-7. https://doi.org/10.1186/1479-5868-5-38

20. Evenson KR, Barakat R, Brown WJ, Dargent MP, Haruna M, Mikkelsen EM, et al. Guidelines for Physical Activity during Pregnancy: Comparisons From Around the World. Am J Lifestyle Med. 2014;8:10221. https://doi.org/10.1177/1559827613498204

21. Lindqvist M, Lindkvist M, Eurenius E, Persson M, Ivarsson A, Mogren I. Leisure time physical activity among pregnant women and its associations with maternal characteristics and pregnancy outcomes. Sex Reprod Health. 2016;9:14-20. https://doi. org/10.1016/j.srhc.2016.03.006

22. Hawkins M, Kim Y, Gabriel KP, Rockette WB, Chasan TL. Sedentary behavior patterns in non-pregnant and pregnant women. Prev Med Rep. 2017;6:97-03. https://doi.org/10.1016/j.pmedr.2017.02.022

23. Connelly M, Brown $H$, van der Pligt P, Teychenne $M$. Modifiable barriers to leisure-time physical activity during pregnancy: a qualitative study investigating first time mother's views and experiences. BMC Pregnancy Childbirth. 2015;15:1-7. https://doi.org/10.1186/ s12884-015-0529-9

24. Thielman J, Rosella L, Copes R, Lebenbaum M, Manson H. Neighborhood walkability: Differential associations with self-reported transport walking and leisure-time physical activity in Canadian towns and cities of all sizes. Prev Med. 2015;77:174-80. https:// doi.org/10.1016/j.ypmed.2015.05.011

25. McEachan RR, Prady SL, Smith G, Fairley L, Cabieses $\mathrm{B}$, Gidlow C, et al. The association between green space and depressive symptoms in pregnant women: Moderating roles of socioeconomic status and physical activity. J Epidemiol Community Health. 
2016;70:253-9. https://doi.org/10.1136/jech-2015205954

26. Vinikoor-Imler LC, Messer LC, Evenson KR, Laraia BA. Neighborhood conditions are associated with maternal health behaviors and pregnancy outcomes. Soc Sci Med. 2011;73:1302-11. https:// doi.org/10.1016/j.socscimed.2011.08.012
27. Giurgescu C, Misra DP, Sealy-Jefferson S, Caldwell $\mathrm{CH}$, Templin TN, Slaughter-Acey JC, et al. The impact of neighborhood quality, perceived stress, and social support on depressive symptoms during pregnancy in African American women. Soc Sci Med. 2015;130:172-80. https://doi.org/10.1016/j. socscimed.2015.02.006

\section{Conflicto de intereses: ninguno declarado.}

